PAPER

Strained band edge characteristics from hybrid density functional theory and empirical pseudopotentials: GaAs, GaSb, InAs and InSb

To cite this article: Asl Çakan et al 2016 J. Phys. D: Appl. Phys. 49085104

View the article online for updates and enhancements.
Related content

Band parameters and strain effects in $\mathrm{ZnO}$ and group-III nitrides

Qimin Yan, Patrick Rinke, M

Winkelnkemper et al.

Electronic and magnetic properties of Ti2O3, Cr2O3, and Fe2O3 calculated by the screened exchange hybrid density

Yunctional Robertson

Reduction of the $\mathrm{Mg}$ acceptor activation energy in GaN, AIN, Al0.83Ga0.17N and MgGa -doping (AIN)5/(GaN)1: the strain effect

Xin-He Jiang, Jun-Jie Shi, Min Zhang et al.

\section{Recent citations}

Complex structure of optical transitions
from the core d-levels of InAs and InSb
crystals 


\title{
Strained band edge characteristics from hybrid density functional theory and empirical pseudopotentials: GaAs, GaSb, InAs and InSb
}

\author{
Aslı Çakan ${ }^{1}$, Cem Sevik ${ }^{2}$ and Ceyhun Bulutay ${ }^{1}$ \\ ${ }^{1}$ Department of Physics, Bilkent University, Bilkent, Ankara 06800, Turkey \\ ${ }^{2}$ Faculty of Engineering, Anadolu University, Eskişehir 26555, Turkey \\ E-mail: bulutay@fen.bilkent.edu.tr
}

Received 18 September 2015, revised 10 December 2015

Accepted for publication 17 December 2015

Published 27 January 2016

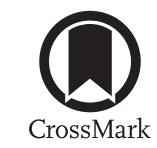

\begin{abstract}
The properties of a semiconductor are drastically modified when the crystal point group symmetry is broken under an arbitrary strain. We investigate the family of semiconductors consisting of GaAs, GaSb, InAs and InSb, considering their electronic band structure and deformation potentials subject to various strains based on hybrid density functional theory. Guided by these first-principles results, we develop strain-compliant local pseudopotentials for use in the empirical pseudopotential method (EPM). We demonstrate that the newly proposed empirical pseudopotentials perform well close to band edges and under anisotropic crystal deformations. Using the EPM, we explore the heavy hole-light hole mixing characteristics under different stress directions, which may be useful in manipulating their transport properties and optical selection rules. The very low 5 Ry cutoff targeted in the generated pseudopotentials paves the way for large-scale EPM-based electronic structure computations involving these lattice mismatched constituents.
\end{abstract}

Keywords: strain in semiconductors, deformation potential, electronic band structure, density functional theory, hybrid functionals, empirical pseudopotential method

(Some figures may appear in colour only in the online journal)

\section{Introduction}

Among group-III-V compound semiconductors crystallizing in the cubic phase, the family of GaAs, GaSb, InAs and InSb span the widest direct bandgap range from $1.51 \mathrm{eV}$ down to $0.235 \mathrm{eV}$ at zero temperature [1]. Their quantum dots such as InAs/GaAs, InSb/GaSb and other combinations have been in the spotlight due to their applications as light emitters, and because of their potential for emerging quantum information technologies [2-5]. For this lattice mismatched family the inevitable overarching theme in their heterostructures is strain. As gradually appreciated over the decades, strain has been a game changer for materials, and especially for semiconductors. As a matter of fact, many of the novel features in these quantum dots, and the very existance of self-assembly are owed to strain [6]. This is also the case for bulk properties. For instance, the nature of bandgap changes from indirect to direct under tensile strain in the case of germanium [7], or higher hole mobilities are attained under strain in group-III-V arsenides, and group-III-V antimonides [8].

Historically, within the context of strain the key concept of deformation potentials were introduced as early as the 1950s by the pioneers of semiconductor physics, Bardeen and Shockley [9] for uniformly strained silicon and germanium, consequently generalized to the multivalley case by Herring and Vogt [10]. The shear deformation parameters related to the conduction band edge for Si was worked out by Sham within the framework of a pseudopotential rigid ion model [11]. In the 1960s, both the conduction and valence band deformation potentials of $\mathrm{Si}$ under hydrostatic and uniaxial 
stress were studied using Kleinman's self-consistent perturbation theory [12]. Subsequently, Ge and GaAs crystals under uniaxial stress were investigated to define the splitting of the valence band and the direct bandgap energy shift within optical reflection measurements by Balslev [13]. Likewise, the effects of uniaxial stress along [0 $\left.0 \begin{array}{ll}0 & 1\end{array}\right],\left[\begin{array}{lll}1 & 1 & 0\end{array}\right]$ and [ [1 11 1] on the electronic structure of Ge, GaAs and Si were investigated by Pollak and Cardona with the calculation of hydrostatic and shear deformation potentials for both conduction and valence bands [14]. The 1970s were stagnant except for Pollak's review [15] and the seminal monograph by Bir and Pikus on strain-induced effects in semiconductors [16]. A revival started with Landolt-Börnstein's experimental data compilation for III-V semiconductors in the 1980s [17]. Afterwards, $a b$ initio theoretical studies abounded [18-25]. As these $a b$ initio calculations suffer from well-known bandgap errors and require high computational cost [26], semi-empirical methods were preferred [27-34]. In particular, O'Reilly using a tight-binding method took into account the crystals under biaxial compression and tension to study the $\left[\begin{array}{lll}0 & 0 & 1\end{array}\right]$ axial deformation potential $b$ in group-III-V semiconductors [35]. He showed that in biaxially strained materials, the heavy hole band could show light-hole type characteristics [30], which have recently been reinstated [25, 33]. Nowadays, this result also has ramifications for quantum information science and technology.

To meet the demand for more up-to-date and reliable semiconductor data Vurgaftman, Meyer and Ram-Mohan (VMR) carried out the most recent compilation, still more than a decade ago [1]. Inevitably, they expressed the deformation potentials as ranges and gave recommendations which are for some materials ambiguous. Notably, there are experimental discrepancies on the $b$ and $d$ biaxial deformation potentials. Therefore, the necessity for reliable deformation potentials for common III-V semiconductors is still a pressing issue within the community.

Over the last decade, density functional theory (DFT) calculations with hybrid functionals have received increasing attention as they offer a remedy for the well-known local density approximation (LDA) failures [26] with the approach by Heyd, Scuseria and Ernzerhof [36]. Hence, it is a wellsuited and reliable method for studies on strained materials. As a matter of fact, using hybrid functionals, the Van de Walle group calculated the shear deformation potentials of $\mathrm{GaN}$ and InN to explore the effect of strain in polarization switching in InGaN/GaN quantum wells [37], and observed pronounced nonlinear dependence on strain for AlN, GaN, InN and $\mathrm{ZnO}$ [38]. For the wurtzite phases of InAs and InP, Hajlaoui et al obtained the deformation potentials and revealed the failure of the quasi-cubic approximation [39]. Interestingly, to the best of our knowledge, a detailed hybrid DFT study for the strained cubic phase GaAs, GaSb, InAs and InSb has not been undertaken.

Our first aim with this work is to extract reliable deformation potentials using the state-of-the-art hybrid DFT computations so as to alleviate the ambiguity in the VMR data [1] over the compounds GaAs, GaSb, InAs and InSb. Moreover, in the light of hybrid DFT computations we develop a new set
Table 1. Comparison of our hybrid DFT and EPM unstrained band structure values with VMR data [1] at $0 \mathrm{~K}$.

\begin{tabular}{|c|c|c|c|c|}
\hline \multirow[b]{2}{*}{ Material } & & \multicolumn{2}{|c|}{ This work } & \multirow[b]{2}{*}{ VMR [1] } \\
\hline & & Hybrid DFT & EPM & \\
\hline & $E_{\text {gap }}$ & 1.36 & 1.51 & 1.52 \\
\hline \multirow[t]{3}{*}{ GaAs } & $\Delta_{0}$ & 0.36 & 0.367 & $0.32-0.36$ \\
\hline & $a_{0}$ & 5.626 & 5.653 & 5.653 \\
\hline & $E_{\text {gap }}$ & 0.81 & 0.812 & $0.811-0.813$ \\
\hline \multirow[t]{3}{*}{$\mathrm{GaSb}$} & $\Delta_{0}$ & 0.72 & 0.723 & $0.749-0.82$ \\
\hline & $a_{0}$ & 6.095 & 6.0959 & 6.0959 \\
\hline & $E_{\text {gap }}$ & 0.34 & 0.41 & $0.41-0.45$ \\
\hline \multirow[t]{3}{*}{ InAs } & $\Delta_{0}$ & 0.38 & 0.388 & $0.37-0.41$ \\
\hline & $a_{0}$ & 6.043 & 6.058 & 6.058 \\
\hline & $E_{\text {gap }}$ & 0.27 & 0.235 & 0.235 \\
\hline \multirow[t]{2}{*}{$\mathrm{InSb}$} & $\Delta_{0}$ & 0.76 & 0.763 & $0.8-0.9$ \\
\hline & $a_{0}$ & 6.457 & 6.479 & 6.479 \\
\hline
\end{tabular}

Note: $E_{\text {gap }}$ is the direct energy gap $(\mathrm{eV}), \Delta_{0}$ represents the valence band spin-orbit splitting $(\mathrm{eV})$, and $a_{0}$ is the equilibrium lattice constant $(\AA)$.

of empirical pseudopotential parameters which can reproduce the band edge hybrid DFT results under various strain profiles, while excluding the nonlocal parts and using a very low 5 Ry energy cutoff in the interest of a reduced computational budget for million-atom structures [40]. Finally, we demonstrate the performance of this empirical pseudopotential parameter set through examining the directional variation of valence band effective masses under uniaxial stress and shed light on the intricate heavy and light-hole mixing characteristics in this material family.

\section{Theory}

\subsection{DFT with hybrid functionals}

Hybrid functionals such as Heyd-Scuseria-Ernzerhof (HSE) combine LDA or generalized gradient approximation (GGA) exchange-correlation functionals with Hartree-Fock (HF) exact exchange [36]. One of the most advantageous features of HSE is to use a conventional local functional instead of a long-range part of the HF term; however, the short-range part is switched to the nonlocal HF potential since the calculation of the long-range part for the localized basis set projected augmented wave (PAW) is troublesome and computationally costly. Conveniently, HSE reduces the high cost of hybrid functionals to within a factor of 2-4 higher than pure DFT functionals, while providing many reliable energy bandgaps for semiconductors [41]. Some of the available hybrid functionals are PBE0 [42], HSE03 [36, 41, 43], HSE06 [44] and HSEsol [45]. For our work we have chosen the HSEsol functional as it is reported to yield satisfying results for small gap semiconductors.

Our $a b$ initio calculations are performed using the Vienna Ab initio Simulation package (VASP) code [46-48]. The PAW pseudopotentials from the standard distribution are incorporated in the calculations $[49,50]$. For electronic exchangecorrelation functional, GGA in its PBEsol parametrization is 
Table 2. Fitted local EPM parameters: form factors and their slopes for cubic Hermite interpolation, symmetric component of the spin-orbit coupling parameter $\lambda_{S}$, and hydrostatic strain parameter $\gamma$, asymptotic cutoff parameters $a_{5}, a_{6}$.

\begin{tabular}{|c|c|c|c|c|c|}
\hline & & & & & \\
\hline & Parameter & GaAs & InAs & $\mathrm{GaSb}$ & $\mathrm{InSb}$ \\
\hline Local form factors & $V_{0}^{s}$ & -0.6421 & -0.5469 & -0.5266 & -0.4246 \\
\hline & $V_{\sqrt{3}}^{s}$ & -0.2350 & -0.2070 & -0.2043 & -0.1990 \\
\hline & $V_{\sqrt{8}}^{s}$ & 0.0150 & 0.0000 & 0.0000 & 0.0115 \\
\hline & $V_{\sqrt{11}}^{s}$ & 0.0729 & 0.0465 & 0.0601 & 0.0334 \\
\hline & $V_{0}^{a}$ & -0.1040 & -0.0880 & -0.0470 & -0.0450 \\
\hline & $V_{\sqrt{3}}^{a}$ & 0.0760 & 0.0540 & 0.0330 & 0.0416 \\
\hline & $V_{\sqrt{4}}^{a}$ & 0.0570 & 0.0466 & 0.0280 & 0.0350 \\
\hline & $V_{\sqrt{11}}^{a}$ & 0.0061 & 0.0070 & 0.0054 & 0.0060 \\
\hline Slopes for PCHIP & $s_{0}^{s}$ & 0.0000 & 0.0000 & 0.0000 & 0.0000 \\
\hline & $s_{\sqrt{3}}^{s}$ & 0.0699 & -0.1760 & -0.1668 & -0.1357 \\
\hline & $s_{\sqrt{8}}^{s}$ & 0.1250 & 0.1250 & 0.1400 & 0.0606 \\
\hline & $s_{\sqrt{11}}^{s}$ & 0.0596 & -0.0062 & -0.0819 & 0.0100 \\
\hline & $s_{0}^{a}$ & 0.0000 & 0.0000 & 0.0000 & 0.0000 \\
\hline & $s_{\sqrt{3}}^{a}$ & 0.0250 & -0.0350 & -0.0500 & -0.0500 \\
\hline & $s_{\sqrt{4}}^{a}$ & -0.1150 & -0.0900 & -0.0400 & -0.0400 \\
\hline & $s_{\sqrt{11}}^{a}$ & -0.0100 & -0.0220 & -0.0300 & -0.0300 \\
\hline Asymptotic parameters & $a_{5}$ & 4.05 & 4.50 & 4.00 & 3.90 \\
\hline & $a_{6}$ & 0.39 & 0.41 & 0.30 & 0.30 \\
\hline Hydrostatic strain parameter & $\gamma$ & -1.7392 & -0.1046 & -2.1285 & -1.4260 \\
\hline Spin-orbit coupling parameter (Ry) & $\lambda_{S}$ & 0.0213 & 0.0205 & 0.0385 & 0.0377 \\
\hline Cutoff energy (Ry) & $E_{\text {cutoff }}$ & 5.00 & 4.85 & 5.00 & 4.85 \\
\hline
\end{tabular}

Note: Refer to text for the units associated with these parameters.

used [51-53]. For the PAW pseudopotentials $d$ orbitals are taken as a valence for the cations, and conventional ones for the anions. The lattice vectors and atomic coordinates are relaxed until the force on each atom is reduced to less than $0.001 \mathrm{eV}$ $\AA^{-1}$ and the total energy is iterated until changes in energy are lower than $10^{-6} \mathrm{eV}$. For each bulk material, $4 \times 4 \times 4$ $k$-grid and $450 \mathrm{eV}$ cutoff energy are used while employing the HSEsol hybrid functional. To impose the hydrostatic and uniaxial stress on the unit cell, we insert the strained lattice vectors. Table 1 shows that the calculated lattice constants are in good agreement with the experimental ones.

\subsection{Empirical pseudopotential method}

Recently, Kim and Fischetti offered local and nonlocal empirical pseudopotential parameters for a number of groupIV and group-III-V semiconductors with 10 Ry cutoff energy [33]. Relying on the success of hybrid DFT electronic structures, we aim to tune the EPM parameters in [33], under the conditions of excluding the nonlocal parts, and using a lower kinetic energy cutoff of $5 \mathrm{Ry}$ to reduce the computational budget.

For arbitrarily strained crystals, the pseudopotential parameters are needed not only at fixed wavenumbers but over a continuum. For this purpose the cubic Hermite interpolation can be used due to its advantage of giving the means to control curve slopes at the desired data points [54]. Accordingly, the local pseudopotential is represented as

$$
V(q)=V_{\mathrm{PCHIP}}(q) \times \frac{1}{2}\left\{\tanh \left[\frac{a_{5}-q^{2}}{a_{6}}\right]+1\right\},
$$

where the $\tanh (\cdot)$ part is introduced for a fast cutoff of the pseudopotential at large wavenumbers, $q$ through the $a_{5}$ and $a_{6}$ fitting parameters; the same values as in [33] are retained, as displayed in table 2, which should be in Hartree atomic units, including $q$ in the tanh part. $V_{\mathrm{PCHIP}}(q)$ represents the piecewise cubic Hermite interpolating polynomial (PCHIP) [54] of the symmetric and antisymmetric local form factors, which consists of potentials and their slopes at certain wavenumbers. For the unit interval $[0,1]$ the PCHIP has the form

$$
\begin{aligned}
V_{\mathrm{PCHIP}}(q)= & \left(2 q^{3}-3 q^{2}+1\right) V_{0}+\left(q^{3}-2 q^{2}+q\right) s_{0} \\
& +\left(-2 q^{3}+3 q^{2}\right) V_{1}+\left(q^{3}-q^{2}\right) s_{1},
\end{aligned}
$$

where $V_{0}$ and $V_{1}\left(s_{0}\right.$ and $\left.s_{1}\right)$ are the potential (slope) values at either end of the interval.

A general drawback of empirical pseudopotentials is that they are frozen in the sense that they lack the self-consistency loop to adapt to changes in the chemical environment such as bond lengths and directions, as would arise under strain. To fix this in the level of hydrostatic strains without a computational overhead, Williamson et al introduced an additional fitting 
Table 3. Effective masses (in free-electron mass, $\left.m_{0}\right)$ at $\Gamma$ point in $k$-space for conduction band $\left(m_{e}^{* \Gamma}\right)$, heavy hole $m_{h h}^{* \Gamma}$, light hole $m_{l h}^{* \Gamma}$ and split-off $m_{s o}^{* \Gamma}$ bands, compared with Kim and Fischetti (KF) [33], and Vurgaftman, Meyer and Ram-Mohan (VMR) data [1].

\begin{tabular}{|c|c|c|c|c|c|c|c|c|c|}
\hline Material & & $m_{e}^{* \Gamma}$ & $m_{h h}^{* \Gamma}\left[\begin{array}{lll}0 & 0 & 1\end{array}\right]$ & $m_{h h}^{* \Gamma}\left[\begin{array}{lll}1 & 1 & 0\end{array}\right]$ & $m_{h h}^{* \Gamma}\left[\begin{array}{lll}1 & 1 & 1\end{array}\right]$ & $m_{l h}^{* \Gamma}\left[\begin{array}{lll}0 & 0 & 1\end{array}\right]$ & $m_{l h}^{* \Gamma}\left[\begin{array}{lll}1 & 1 & 0\end{array}\right]$ & $m_{l h}^{* \Gamma}\left[\begin{array}{lll}1 & 1 & 1\end{array}\right]$ & $m_{s o}^{* \Gamma}$ \\
\hline \multirow{3}{*}{ GaAs } & This work & 0.082 & 0.439 & 0.845 & 1.143 & 0.111 & 0.099 & 0.096 & 0.218 \\
\hline & $\mathrm{KF}$ & 0.082 & 0.382 & 0.696 & 0.903 & 0.106 & 0.094 & 0.091 & 0.206 \\
\hline & VMR & 0.063 & 0.388 & 0.658 & 0.920 & 0.089 & 0.081 & 0.079 & $0.33-0.388$ \\
\hline \multirow{3}{*}{$\mathrm{GaSb}$} & This work & 0.054 & 0.363 & 0.724 & 1.027 & 0.066 & 0.060 & 0.059 & 0.20 \\
\hline & $\mathrm{KF}$ & 0.049 & 0.289 & 0.534 & 0.712 & 0.056 & 0.052 & 0.050 & 0.19 \\
\hline & VMR & 0.041 & 0.23 & - & 0.57 & - & 0.05 & - & 0.14 \\
\hline \multirow{3}{*}{ InAs } & This work & 0.030 & 0.433 & 0.814 & 1.127 & 0.038 & 0.036 & 0.036 & 0.127 \\
\hline & $\mathrm{KF}$ & 0.026 & 0.31 & 0.547 & 0.720 & 0.032 & 0.03 & 0.03 & 0.109 \\
\hline & VMR & 0.023 & 0.39 & 0.98 & 0.757 & 0.042 & 0.041 & 0.014 & $0.09-0.15$ \\
\hline \multirow{3}{*}{$\mathrm{InSb}$} & This work & 0.022 & 0.357 & 0.714 & 1.049 & 0.024 & 0.023 & 0.023 & 0.172 \\
\hline & $\mathrm{KF}$ & 0.017 & 0.304 & 0.534 & 0.705 & 0.019 & 0.018 & 0.018 & 0.155 \\
\hline & VMR & 0.014 & 0.26 & - & 0.68 & 0.015 & 0.015 & - & 0.19 \\
\hline
\end{tabular}

parameter $\gamma$ so that a hydrostatic strain-dependent pseudopotential is formed as [31]

$$
V(q ; \epsilon)=V(q)\left[1+\gamma \epsilon_{\mathrm{H}}\right],
$$

where $\epsilon_{\mathrm{H}}=\epsilon_{x x}+\epsilon_{y y}+\epsilon_{z z}$ refers to the hydrostatic (volumetric) strain.

For an even better strain performance, we combine Kim and Fischetti local EPM form factors [33] with the hydrostatic strain parameter, $\gamma[31]$. Then, we optimize this set including the symmetric spin-orbit coupling parameter $\lambda_{S}$ [31, 55], choosing the target values as the experimental bandgaps, and our hybrid DFT deformation potentials, and spin-orbit splittings. Note that in contrast to [33], we fit EPM to the zerotemperature bandgaps shown in table 1 . Table 2 presents our EPM parameters at standard wave numbers of the zinc-blende structure: $0, \sqrt{3}, \sqrt{4}, \sqrt{8}, \sqrt{11}$, all in units of $2 \pi / a_{0}$, where $a_{0}$ is the unstrained lattice constant of the crystal; the form factors and their slopes are set to zero beyond a high value, $q^{2}>50$. Regarding the associated units for these parameters, other than the tanh part, which was separately mentioned above, $V(q)$ in (1) comes out in Ry when wavenumber $q$ is used in units of $2 \pi / a_{0} . V^{s, a}(q=0)$ are adjusted to the $a b$ initio natural band offsets under unstrained conditions offered in [56]. It can be seen from table 3 that our local EPM conduction and valence band edge effective mass values are in reasonable agreement with [33], and the latter is in fair agreement with the VMR data [1].

\section{Results}

\subsection{Hybrid DFT results}

The hybrid functional DFT band structures for unstrained $\mathrm{GaAs}$, GaSb, InAs and InSb including the spin-orbit interaction are displayed in figure 1. For these direct bandgap compounds, the computed bandgap values are within $10 \%$ agreement with the experimental values in table 1 . If desired, further improvement is possible by slightly adjusting the so-called range separation parameter of the hybrid functionals [57].
Next, from hybrid DFT calculations we work out the deformation potentials $a_{\text {gap }}, b$ and $d$ under the relevant stress conditions: hydrostatic, uniaxial stress along [l $\left.\begin{array}{lll}0 & 0 & 1\end{array}\right]$ and $\left[\begin{array}{lll}1 & 1 & 1\end{array}\right]$, respectively [58]. Our results are listed in table 4 together with VMR [1], and other representative data from the literature. Notably, in some cases the VMR ranges and/or suggested values deviate substantially from the hybrid DFT results.

An interesting strain condition is when the valence band maximum (VBM) is no longer a pure $\left|\frac{3}{2}, \frac{3}{2}\right\rangle$ state, namely a heavy hole band, but rather is dominated by the light hole character of the $\left|\frac{3}{2}, \frac{1}{2}\right\rangle$ state. This occurs for the uniaxial stress applied along the [ [ $\left.\begin{array}{lll}0 & 0 & 1\end{array}\right]$ and [ [ $\left.\begin{array}{lll}1 & 1 & 1\end{array}\right]$ directions provided that the biaxial strain $\epsilon_{\mathrm{B}} \equiv \epsilon_{z z}-\left(\epsilon_{x x}+\epsilon_{y y}\right) / 2<0$, and $\epsilon_{x y}<0$ conditions hold, respectively. Figure 2 illustrates the variation of the energy splitting between the two uppermost valence bands, VBM and VBM-2, as a function of the negative biaxial $\epsilon_{\mathrm{B}}$ and off-diagonal $\epsilon_{x y}$ strains under uniaxial stresses along the [ll $\left.\begin{array}{lll}0 & 0 & 1\end{array}\right]$ or $\left[\begin{array}{lll}1 & 1 & 1\end{array}\right]$ directions, respectively. A cation-based grouping is clearly visible, that is, GaAs and GaSb behave in a similar way, as do InAs and InSb. Moreover, even though a linear trend is manifested under the [ $\left[\begin{array}{lll}0 & 0 & 1\end{array}\right]$ uniaxial stress for strains exceeding $6 \%$, nonlinear behaviour can be observed at smaller strains in InAs and InSb under the [ $\left.\begin{array}{lll}1 & 1 & 1\end{array}\right]$ uniaxial stress. The nature of the uppermost valence bands will be further analysed below with regard to the directional characteristics of the effective mass.

\subsection{EPM band edge and effective mass characteristics under strain}

We would like to contrast the band edge performance of the EPM as described by the parameters contained in table 2 with the hybrid DFT results in the presence of various strain conditions. First, we start with the hydrostatic strain, and compare in figure 3 the shift of the direct bandgap from its unstrained value. Since InSb and InAs become metallic, only a limited tensile strain is applied, whereas on the compressive side up to $12 \%$ hydrostatic strain is considered. The agreement of the EPM with the hybrid DFT results are seen to be 

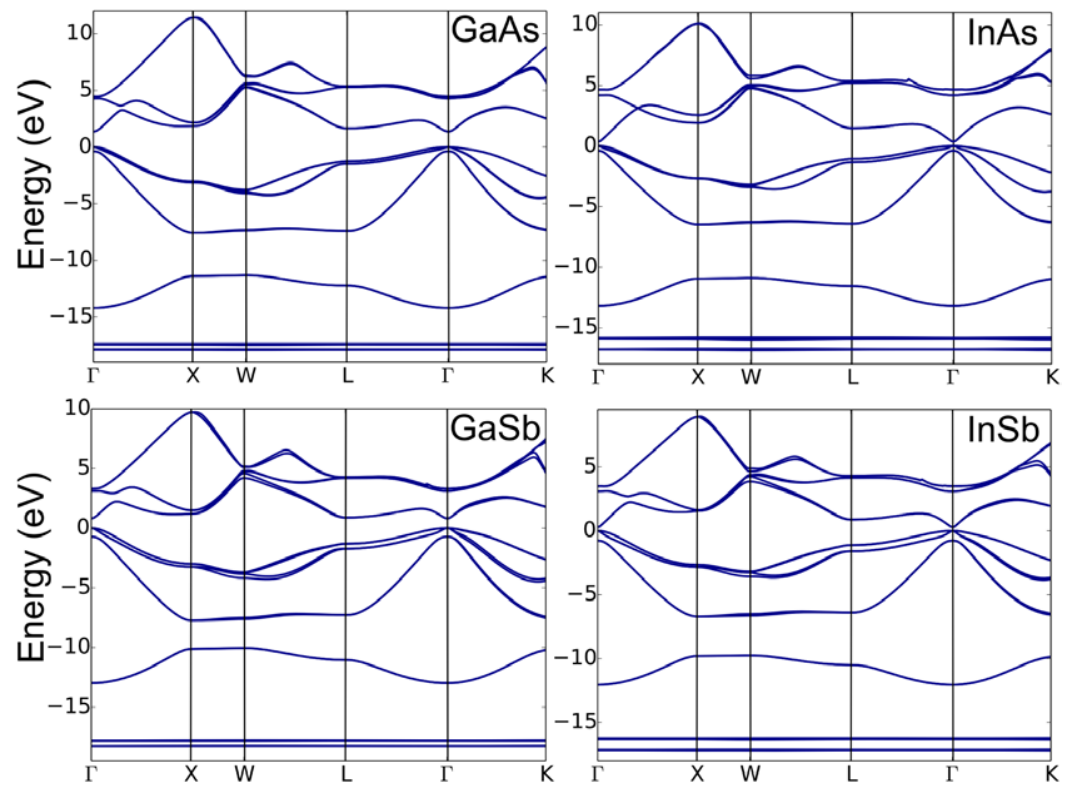

Figure 1. Full zone band structures for unstrained GaAs, GaSb, InAs and InSb obtained using hybrid functional DFT. The valence band maximum is set to zero energy level separately for each material.

Table 4. Deformation potentials $a_{\text {gap }}, b$ and $d$ in $\mathrm{eV}$ units. Here, the EPM values are fitted to the hybrid DFT results.

\begin{tabular}{|c|c|c|c|c|}
\hline \multirow[b]{2}{*}{ Material } & & \multirow{2}{*}{$\begin{array}{l}\text { This work } \\
\text { Hybrid DFT \& EPM }\end{array}$} & \multicolumn{2}{|r|}{ Literature } \\
\hline & & & $\mathrm{VMR}^{\mathrm{a}}$ & Others \\
\hline \multirow{3}{*}{ GaAs } & $a_{\text {gap }}$ & -8.69 & {$[-20.4,-6.5]$} & $-8.33^{\mathrm{d}},-8.76^{\mathrm{e}},-8.33^{\mathrm{h}},-8.44^{\mathrm{j}},-7.25^{\mathrm{k}}$ \\
\hline & $b$ & -2.13 & {$[-3.9,-1.6]$} & $-2.0^{\mathrm{c}, \mathrm{d}},-1.7^{\mathrm{f}},-1.9^{\mathrm{h}, \mathrm{e}},-2.79^{\mathrm{b}, \mathrm{h}}$ \\
\hline & $d$ & -4.77 & {$[-6.0,-2.7]$} & $-4.23^{\mathrm{h}, \mathrm{e}},-4.5^{\mathrm{c}},-4.77^{\mathrm{g}},-7.5^{\mathrm{b}}$ \\
\hline \multirow{3}{*}{$\mathrm{GaSb}$} & $a_{\text {gap }}$ & -8.44 & -8.3 & $-7.64^{\mathrm{h}},-7.01^{\mathrm{k}}$ \\
\hline & $b$ & -2.23 & -1.6 & $-1.6^{\mathrm{b}},-1.9^{\mathrm{f}},-2.0^{\mathrm{c}},-2.3^{\mathrm{g}}$ \\
\hline & $d$ & -5.0 & -3.98 & $-3.98^{\mathrm{g}},-4.7^{\mathrm{c}},-4.8^{\mathrm{i}},-5.0^{\mathrm{b}}$ \\
\hline \multirow{3}{*}{ InAs } & $a_{\text {gap }}$ & -5.95 & {$[-16.9,-6.08]$} & $-6.12^{\mathrm{e}},-6.08^{\mathrm{h}},-4.93^{\mathrm{k}}$ \\
\hline & $b$ & -1.76 & {$[-5.9,-1.0]$} & $-1.72^{\mathrm{b}},-1.55^{\mathrm{h}, \mathrm{e}},-1.7^{\mathrm{f}, \mathrm{d}},-1.8^{\mathrm{c}},-2.33^{\mathrm{g}}$ \\
\hline & $d$ & -4.25 & {$[-8,-2.57]$} & $-3.3^{\mathrm{b}},-3.6^{\mathrm{c}},-3.1^{\mathrm{h}, \mathrm{e}},-3.83^{\mathrm{g}}$ \\
\hline \multirow{3}{*}{$\mathrm{InSb}$} & $a_{\text {gap }}$ & -6.67 & -7.2 & $-6.53^{\mathrm{h}},-5.60^{\mathrm{k}}$ \\
\hline & $b$ & -1.88 & -2.0 & $-2.3^{\mathrm{b}},-2.0^{\mathrm{c}, \mathrm{g}},-1.9^{\mathrm{f}}$ \\
\hline & $d$ & -4.62 & -4.7 & $-4.8^{\mathrm{c}},-5.2^{\mathrm{b}}$ \\
\hline
\end{tabular}

${ }^{\mathrm{a}}[1],{ }^{\mathrm{b}}[33],{ }^{\mathrm{c}}[17],{ }^{\mathrm{d}}[31],{ }^{\mathrm{e}}[32],{ }^{\mathrm{f}}[30],{ }^{\mathrm{g}}[29],{ }^{\mathrm{h}}[18],{ }^{\mathrm{i}}[59],{ }^{\mathrm{j}}[23],{ }^{\mathrm{k}}[20]$.

excellent up to $6 \%$ compressive strain, beyond which a deviation is observed, predominantly for GaAs. In practical considerations, as within this family, GaAs has the smallest lattice constant and the widest bandgap; it is generally used as the host matrix material, and therefore it seldom experiences such strain levels.

Figures 4 and 5 extend the comparison for all four materials to uniaxial stress along [l $\left.\begin{array}{lll}0 & 0 & 1\end{array}\right]$ and [ $\left[\begin{array}{lll}1 & 1 & 1\end{array}\right]$, respectively. For $\left[\begin{array}{lll}0 & 0 & 1\end{array}\right]$ stress, the strain tensor has the components $\epsilon_{x x}=\epsilon_{y y}=0.01, \epsilon_{z z}=-0.02$ with the other off-diagonal entries being zero, which corresponds to a biaxial strain of $\epsilon_{\mathrm{B}}=-0.03$. The [ $\left[\begin{array}{lll}1 & 1 & 1\end{array}\right]$ stress is reflected by the off-diagonal strain components of -0.01 and with all diagonal entries being zero. As we fit the EPM directly to the experimental values (see table 1) a slight bandgap discrepancy is discernible for $\mathrm{GaAs}$ and $\mathrm{InSb}$ in these figures. Overall, it can be observed that the band edge behaviour of the EPM agrees well with the HSEsol results within an energy span of at least a few hundred millielectronvolts of the respective band edge. The reproduction of the crossings of the valence bands along the stress directions is particularly crucial.

In addition to these crystallographic directions of [l $\left.\begin{array}{lll}0 & 0 & 1\end{array}\right]$

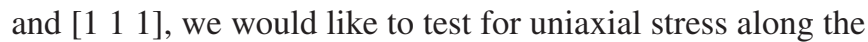

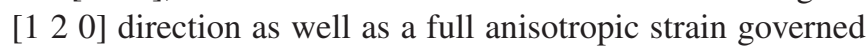
by the tensors

$$
\begin{aligned}
\epsilon_{[120]} & =\left[\begin{array}{ccc}
-0.001455 & 0.00842 & 0 \\
0.00842 & 0.02163 & 0 \\
0 & 0 & -0.00915
\end{array}\right], \\
\epsilon_{\text {full }} & =\left[\begin{array}{ccc}
0.01 & 0.003 & 0.007 \\
0.003 & 0.01 & 0.02 \\
0.007 & 0.02 & -0.015
\end{array}\right] .
\end{aligned}
$$



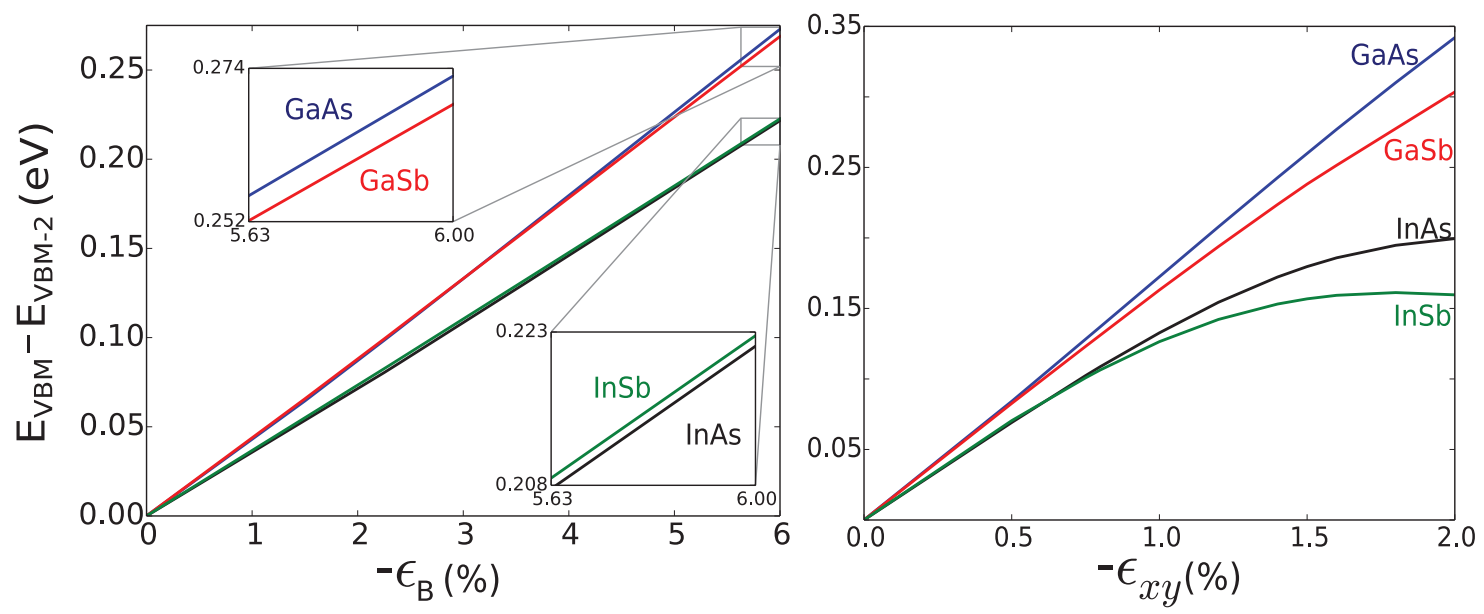

Figure 2. Energy splitting between the VBM and VBM-2 (which is the band just below the VBM in the presence of spin degrees of freedom) obtained using hybrid functional DFT for negative $\epsilon_{\mathrm{B}} \equiv \epsilon_{z z}-\left(\epsilon_{x x}+\epsilon_{y y}\right) / 2$ and negative $\epsilon_{x y}$ strains under uniaxial stress along [0 $\left.\begin{array}{lll}0 & 1\end{array}\right]$ (left), and along [ $\left[\begin{array}{lll}1 & 1 & 1\end{array}\right]$ (right), respectively.

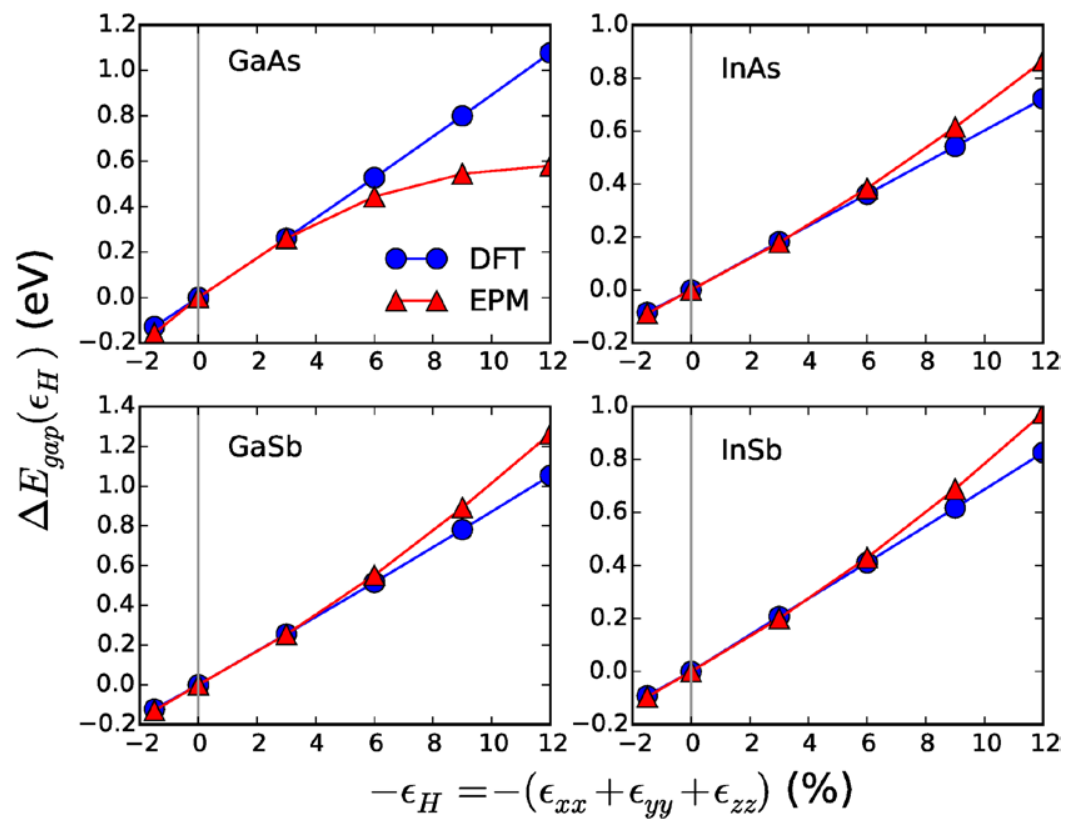

Figure 3. Comparison of hybrid functional DFT versus EPM for the shift of the direct bandgap from their unstrained values as a function of negative hydrostatic strain.

The comparison for these cases for GaAs shown in figure 6 assures that the developed EPM fitting faithfully represents the HSEsol results under arbitrary strain profiles. However, it should be noted that away from the band edges, the EPM starts to deviate from the hybrid DFT, as seen in figures 4-6. Thus, this set is specifically suitable for optical and excitonic characteristics around the $\Gamma$ point of the valence and conduction bands under diverse strain conditions.

Having established the band edge performance of the EPM set, we turn to the valence band effective mass characteristics in the absence and presence of strain. As we have seen in figure 2, deforming the crystal symmetry of the zincblend structure through stress removes the degeneracy at the VBM. Now we would like to explore the nature of the bands, and in particular the directional mixing traits between the heavy and light hole bands with respect to the unstrained case. Figure 7 displays the directional variation of the effective mass of the top three valence bands, as computed using the EPM. Because of further band couplings under strain, the effective masses change sign, therefore we prefer to plot the inverses of the effective masses. The top panel contains the unstrained case, where the VBM is the heavy hole band for all directions, and manifests the well-known warping behaviour more distinctly than the light hole band [60]. The centre and bottom panels in figure 7 show the cases under uniaxial stress along the [0 $\left[\begin{array}{lll}0 & 0 & 1\end{array}\right]$ and [ $\left[\begin{array}{lll}1 & 1 & 1\end{array}\right]$ directions, respectively. The underlying strain tensors are the same as those for figures 4 and 5, as quoted above. For either case, the VBM exhibits a light-hole character along the stress direction, whereas in perpendicular to the stress direction the roles are swapped. 

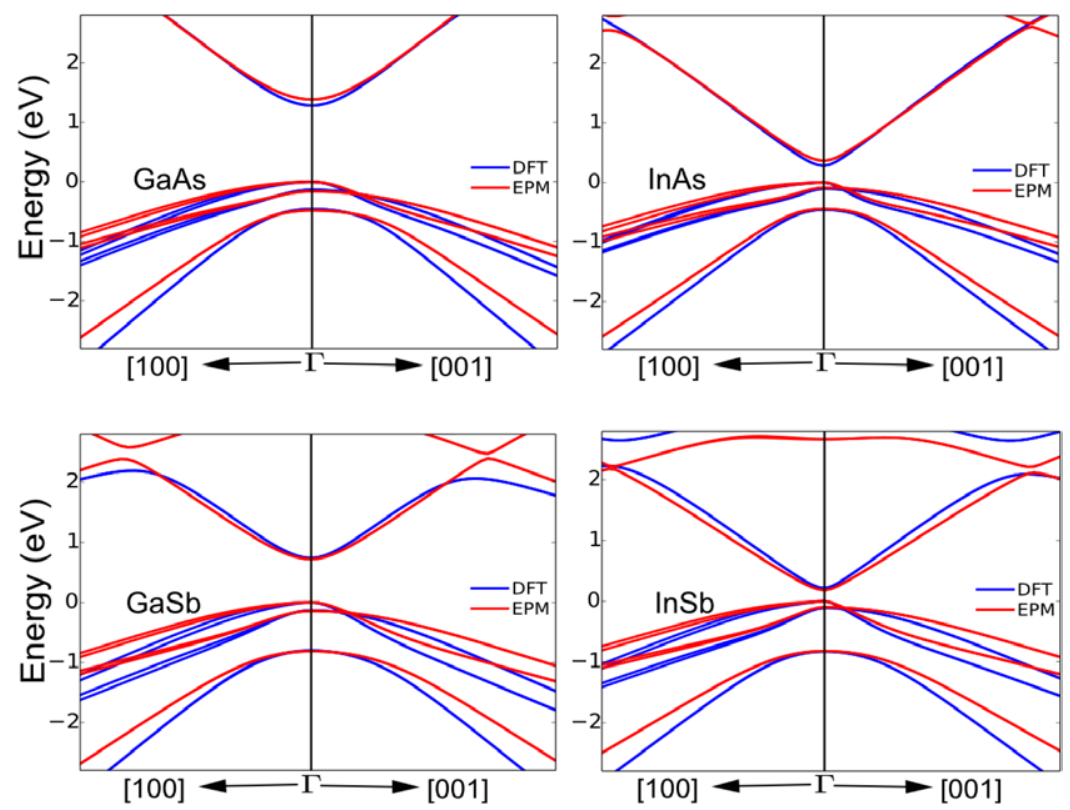

Figure 4. Band edge characteristics of hybrid functional DFT and EPM under uniaxial stress along $\left[\begin{array}{lll}0 & 0 & 1\end{array}\right]$. For each material the VBM is set to zero energy level. The associated strain tensor is provided in the text.
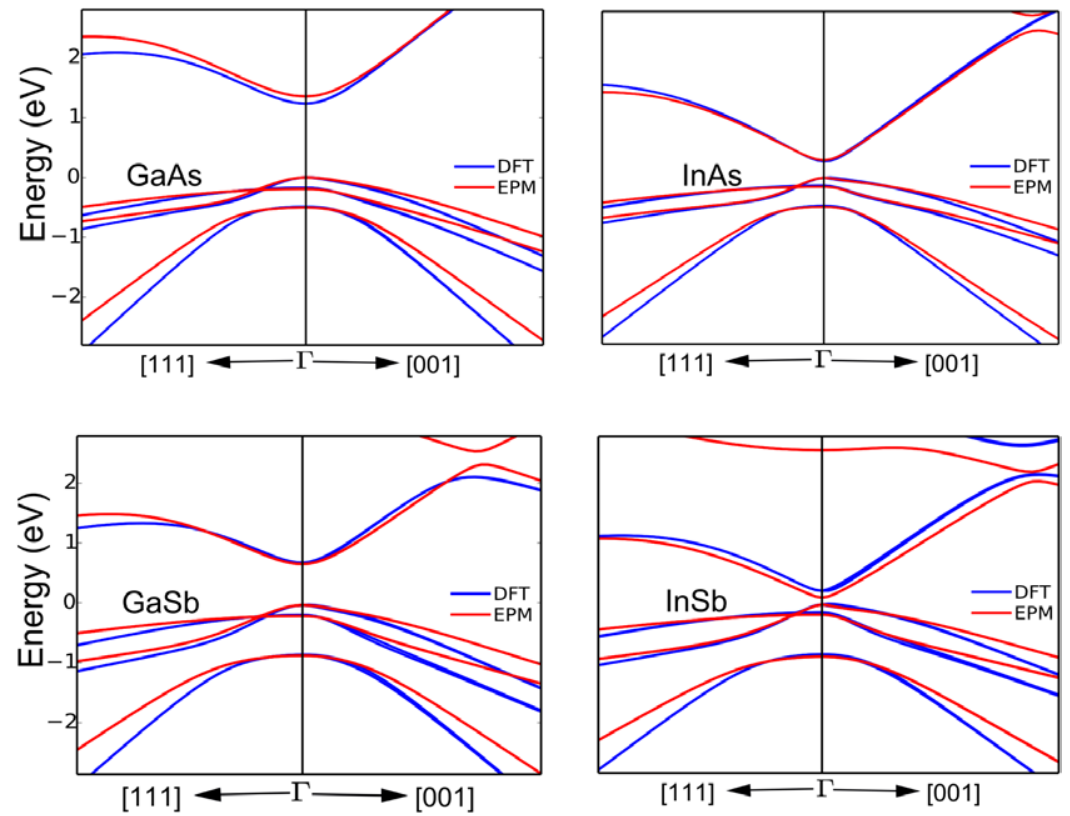

Figure 5. The same as the previous figure, but under uniaxial stress along [llllll.
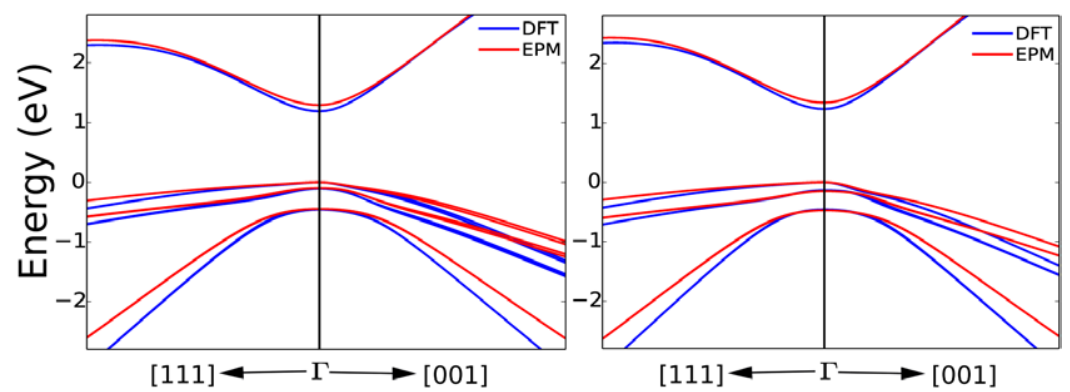

Figure 6. Band edge characteristics for GaAs of hybrid functional DFT and EPM for uniaxial stress along [1 2 0] (left), and for full anisotropic strain (right). The associated strain tensors are provided in the text. 

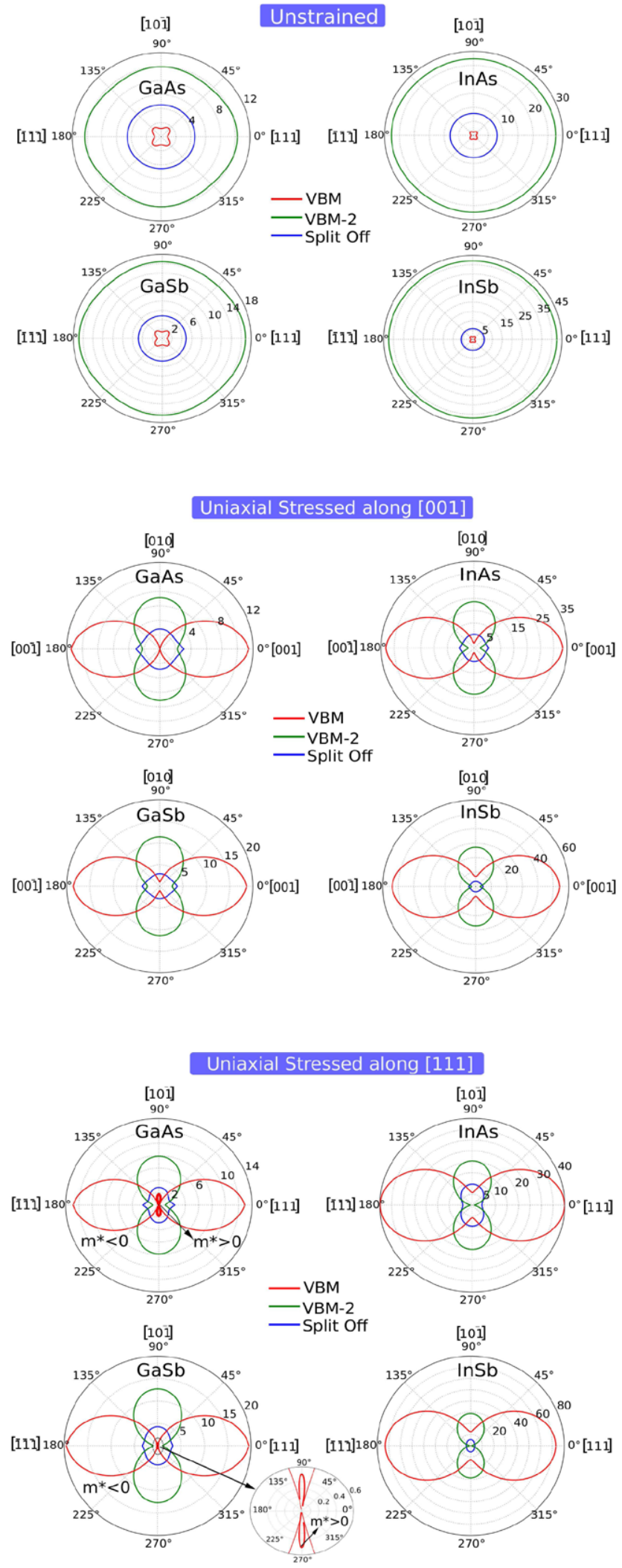

Figure 7. Directional variation of the EPM valence band inverse effective masses, $m_{0} /\left|m^{*}\right|$ for unstrained (top), and uniaxially

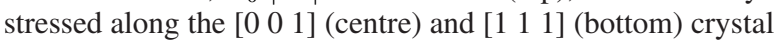
directions. The inset and thick red lines highlight the angular regions where the VBM acquires an electron-like positive effective mass.
As a matter of fact, for the [lll $\left.\begin{array}{lll}1 & 1 & 1\end{array}\right]$ uniaxial stress in GaAs and $\mathrm{GaSb}$, perpendicular to the stress direction the VBM even changes sign and behaves like an electron band, see the inset in the bottom panel. The split-off band, being energetically remote from the upper two valence bands, preserves its isotropic behaviour more or less in all the cases considered above. In general terms, our analysis supports the recently measured higher hole mobility in group-III-V arsenides, and group-III-V antimonides under strain [8], even though there is a non-trivial directional mixing between heavy and light hole bands.

\section{Conclusions}

For the technologically important semiconductors of GaAs, $\mathrm{GaSb}$, InAs and InSb, hybrid DFT calculations are employed to extract accurate band edge deformation potentials. Relying on these first-principles data, we offer a new EPM parametrization with superior performance under arbitrarily strained conditions. Through these EPM band structures, we demonstrate how the valence bands change character as a function of orientation under uniaxial stresses. This reveals that the VBM shows a light hole nature along the stress direction while displaying heavy hole behaviour towards perpendicular directions. Since this has implications in the optical selection rules and spin injection, it may be of importance for spintronics or other quantum technologies $[4,5]$. Given the reliability of our scheme, we believe that through a similar hybrid DFT study, the conduction band shear deformation potentials of the higher-lying degenerate valleys which are beyond the scope of this work can also be extracted.

\section{Acknowledgments}

We would like to thank TÜBİTAK, The Scientific and Technological Research Council of Turkey for their financial support through project No. 112T178. The numerical calculations reported in this paper were partially performed at TÜBİTAK ULAKBIM, High Performance and Grid Computing Center (TRUBA resources).

\section{References}

[1] Vurgaftman I, Meyer J R and Ram-Mohan L R $2001 \mathrm{~J}$. Appl. Phys. 89 5815-75

[2] Alphandry E, Nicholas R J, Mason N J, Zhang B, Möck P and Booker G R 1999 Appl. Phys. Lett. 74 2041-3

[3] He L, Bester G and Zunger A 2004 Phys. Rev. B 70235316

[4] Zieliski M 2013 J. Phys.: Condens. Matter 25465301

[5] Huo Y H et al 2014 Nat. Phys. 10 46-51

[6] Stangl J, Holý V and Bauer G 2004 Rev. Mod. Phys. 76 725-83

[7] Cannon D D, Liu J, Ishikawa Y, Wada K, Danielson D T, Jongthammanurak S, Michel J and Kimerling L C 2004 Appl. Phys. Lett. 84 906-8 
[8] Nainani A, Bennett B R, Brad Boos J, Ancona M G and Saraswat K C 2012 J. Appl. Phys. 111103706

[9] Bardeen J and Shockley W 1950 Phys. Rev. 80 72-80

[10] Herring C and Vogt E 1956 Phys. Rev. 101 944-61

[11] Sham L J 1963 Proc. Phys. Soc. 81934

[12] Goroff I and Kleinman L 1963 Phys. Rev. 132 1080-4

[13] Balslev I 1967 Solid State Commun. 5 315-7

[14] Pollak F H and Cardona M 1968 Phys. Rev. 172 816-37

[15] Pollak F H 1973 Surf. Sci. 37 863-95

[16] Bir G L and Pikus G E 1974 Symmetry and Strain-induced Effects in Semiconductors (New York: Wiley)

[17] Madelung O, Schulz M, Weiss H and Hellwege K H (ed) 1982 Crystal and Solid State Physics (Landolt-Börnstein: Numerical Data and Functional Relationships in Science andTechnology vol 17) (Berlin: Springer)

[18] Van de Walle C G 1989 Phys. Rev. B 39 1871-83

[19] Van de Walle C G and Martin R M 1989 Phys. Rev. Lett. 62 2028-31

[20] Wei S H and Zunger A 1999 Phys. Rev. B 60 5404-11

[21] Li Y H, Gong X G and Wei S H 2006 Appl. Phys. Lett. 88042104

[22] Li Y H, Gong X G and Wei S H 2006 Phys. Rev. B 73245206

[23] Cheiwchanchamnangij T and Lambrecht W R L 2011 Phys. Rev. B 84035203

[24] Kadantsev E S, Zieliński M and Hawrylak P 2012 Phys. Rev. B 86085411

[25] Cárdenas J R and Bester G 2012 Phys. Rev. B 86115332

[26] Martin R M 2004 Electronic Structure: Basic Theory and Practical Methods (Cambridge: Cambridge University Press)

[27] Blacha A, Presting H and Cardona M 1984 Phys. Status Solidi b 126 11-36

[28] Cardona M and Christensen N E 1987 Phys. Rev. B 35 6182-94

[29] Priester C, Allan G and Lannoo M 1988 Phys. Rev. B 37 8519-22

[30] Silver M, Batty W, Ghiti A and O'Reilly E P 1992 Phys. Rev. B 46 6781-88

[31] Williamson A J, Wang L W and Zunger A 2000 Phys. Rev. B 62 12963-77

[32] Niquet Y M 2006 Phys. Rev. B 74155304

[33] Kim J and Fischetti M V 2010 J. Appl. Phys. 108013710

[34] Wang J, Gong M, Guo G C and He L 2012 J. Phys.: Condens. Matter 24475302

[35] O’Reilly E P 1986 Semicond. Sci. Technol. 1128
[36] Heyd J, Scuseria G E and Ernzerhof M 2003 J. Chem. Phys. 118 8207-15

[37] Yan Q, Rinke P, Scheffler M and Van de Walle C G 2010 Appl. Phys. Lett. 97181102

[38] Yan Q, Rinke P, Winkelnkemper M, Qteish A, Bimberg D, Scheffler M and de Walle C G V 2011 Semicond. Sci. Technol. 26014037

[39] Hajlaoui C, Pedesseau L, Raouafi F, CheikhLarbi F B, Even J and Jancu J M 2013 J. Phys. D: Appl. Phys. 46505106

[40] Wang L W and Zunger A 1999 Phys. Rev. B 59 15806-18

[41] Heyd J and Scuseria G E 2004 J. Chem. Phys. 121 1187-92

[42] Adamo C and Barone V 1999 J. Chem. Phys. 110 6158-70

[43] Heyd J, Scuseria G E and Ernzerhof M 2006 J. Chem. Phys. 124219906

[44] Krukau A V, Vydrov O A, Izmaylov A F and Scuseria G E 2006 J. Chem. Phys. 125224106

[45] Schimka L, Harl J and Kresse G 2011 J. Chem. Phys. 134024116

[46] Kresse G and Hafner J 1993 Phys. Rev. B 47 558-61

[47] Kresse G and Hafner J 1994 Phys. Rev. B 49 14251-69

[48] Kresse G and Furthmuller J 1996 Comput. Mater. Sci. 6 15-50

[49] Blöchl P E 1994 Phys. Rev. B 50 17953-79

[50] Kresse G and Joubert D 1999 Phys. Rev. B 59 1758-75

[51] Perdew J P, Burke K and Ernzerhof M 1996 Phys. Rev. Lett. 77 3865-68

[52] Perdew J P, Ruzsinszky A, Csonka G I, Vydrov O A, Scuseria G E, Constantin L A, Zhou X and Burke K 2008 Phys. Rev. Lett. 100136406

[53] Perdew J P, Ruzsinszky A, Csonka G I, Vydrov O A, Scuseria G E, Constantin L A, Zhou X and Burke K 2009 Phys. Rev. Lett. 102039902

[54] Fritsch F and Carlson R 1980 SIAM J. Numer. Anal. 17 238-46

[55] Wang L W, Kim J and Zunger A 1999 Phys. Rev. B 59 5678-87

[56] Li Y H, Walsh A, Chen S, Yin W J, Yang J H, Li J, Da Silva J L F, Gong X G and Wei S H 2009 Appl. Phys. Lett. 94212109

[57] Moussa J E, Schultz P A and Chelikowsky J R 2012 J. Chem. Phys. 136204117

[58] Yu P Y and Cardona M 2005 Fundamentals of Semiconductors, Physics and Materials Properties (Berlin: Springer)

[59] Noack R A, Rühle W and Morgan T N 1978 Phys. Rev. B 18 6944-56

[60] Hensel J C and Feher G 1963 Phys. Rev. 129 1041-62 